\title{
LAS TECNOLOGÍAS EN LOS HÁBITOS ALIMENTICIOS SALUDABLES DE LOS ESTUDIANTES DE ADMINISTRACIÓN DE EMPRESAS
}

\section{TECHNOLOGIES IN THE HEALTHY EATING HABITS OF BUSINESS STUDENTS}

\author{
Yuliana Ortiz Torrado ${ }^{1}$ \\ Carlos Alberto Pacheco Sánchez ${ }^{2}$ \\ ${ }^{1}$ Corporación de Gestión Empresarial, Investigativa y de proyectos, Ocaña, Colombia \\ https://orcid.org/0000-0003-0281-1176,E-mail: ortiztorradojuliana@gmail.com \\ ${ }^{2}$ Corporación de Gestión Empresarial, Investigativa y de proyectos, Ocaña, Colombia \\ https://orcid.org/0000-0002-9369-582X,E-mail: carpasa6@gmail.com
}

Resumen: El artículo científico se enfoca en cuán incidentes son las tecnologías de la información y la comunicación para promover una cultura alimentaria saludable y balanceada que asegure un mejor estado de salud y un buen estilo de vida de los estudiantes, por ello, el objetivo de la investigación es conocer el uso de las tecnologías en la cultura alimentaria. Por tal motivo, el estudio cuantitativo se aplicó la técnica de la encuesta a 300 estudiantes de la Universidad Francisco de Paula Santander Ocaña; Se concluye que la comunidad estudiantil desconoce y no tiene interés sobre las herramientas de sitio web para recibir formación sobre los aspectos nutricionales.

Palabras claves: Cultura alimentaria, Estudiantes, Nutrición, Tecnologías.

\begin{abstract}
The scientific article focuses on how information and communication technologies can promote a healthy and balanced food culture that ensures a better state of health and a good lifestyle for students. Therefore, the objective of the research is to understand the use of technologies in food culture. For this reason, the quantitative study applied the survey technique to 300 students at the Francisco de Paula Santander Ocaña University. It showed that the student community does not know and is not interested in the website tools to receive training on nutritional aspects.
\end{abstract}

Keywords: Food culture, Students, Nutrition, Technologies. 


\section{Introducción}

Llevar un buen estilo de vida junto con una alimentación saludable es un factor que permite al ser humano en sus etapas de crecimiento y desarrollo tener una vida saludable y sana, obteniendo defensas fuertes que combatan posibles enfermedades, de manera que, para lograr una alimentación de calidad es indispensable la disciplina, el orden y compromiso.

En un sentido amplio, para mantener la salud, el crecimiento y el desarrollo de las personas es transcendental tener una cantidad suficiente de diferentes macro y micronutrientes como para cubrir la mayoría de las necesidades fisiológicas. Estas necesidades están influenciadas por distintos factores como el sexo, la edad, el estado fisiológico (embarazo, lactancia, crecimiento), la composición corporal, la actividad física y las características específicas de cada individuo (Bellido, 2006).

Por tanto, cuando una persona o un grupo de personas eligen llevar un modo de vida "diferente" en cuanto a la alimentación, existe mayor probabilidad de que su salud y su cuerpo se mantengan en buenas condiciones y de ese modo prevenir futuras enfermedades que puedan ocasionarles algún percance.

La alimentación es un procesamiento que va desde la gestación hasta la vejez, el ser humano debe, para poder subsistir, de alguna manera alimentarse. La diferencia está en cómo lo hacen. Si bien es conocido, en su gran mayoría las personas prefieren no tener cuidado de lo que comen, tal vez porque no les interesa, no tienen conocimiento para hacerlo y es ahí donde suelen aparecer muchas deficiencias y enfermedades de salud.
Las entidades virtuales dedicadas a dicha actividad económica, deben proponer estrategias claves para que la sociedad tenga una excelente alimentación y un mejor estilo de vida, es decir, lograr que día a día las personas apropien rutinas saludables sin dejar de lado el ejercicio y mejores prácticas, esto con el fin de crear en las mentes de ellos un cambio en la manera de alimentarse y de verse $\mathrm{y}$ sentirse mejor (estimulo motivacional).

El reto hoy día, es lograr una vida con resiliencia y con la convicción de tener una alimentación balanceada. No obstante, en la actualidad el hábito de comer saludablemente es practicada por una pequeña población en el mundo, dado que, ahora es más satisfactorio tomar la elección de adquirir comida rápida (flexibilidad) a pesar de que regularmente no constituye un beneficio para el organismo humano. (Ortiz, 2017) afirma:

\footnotetext{
Comer es una necesidad primaria que todos los seres requieren para vivir, siendo el alimento la necesidad y los nutrientes los requerimientos para vivir. Alimento y nutrientes son diferentes conceptualmente, ya que el primero es lo que vemos y nos atrae para comer, mientras que los nutrientes no los vemos, forman parte intrínseca de los alimentos, que permite nutrirnos. (p.4)
}

Seguidamente y partiendo de los requerimientos en cuanto a lo que necesita el cuerpo humano para alimentarse llega la necesidad de hacerlo correctamente (percepción adecuada de nutrición), es decir, es beneficioso que se haga de modo adecuado para tener sistema inmune que proteja nuestro organismo de enfermedades y permita llevar una vida tranquila (Sin patologías crónicas como obesidad, cáncer, diabetes, colesterol, etc.). Agregado a esto, las tecnologías hoy en día brindan la facilidad de conocer más a fondo que alimentos se necesitan y sus métodos de preparación, pues el avance de las 
tecnologías desde páginas, aplicaciones, canales y demás, aportan de manera dinámica las orientaciones claves para el consumo correcto de alimentos.

La salud de los jóvenes se ve principalmente afectada por la poca preocupación sobre lo que consumen diariamente y el descuido a eso mismo. Ahora, el periodo de estudios universitarios suele ser el momento en que los estudiantes asumen por primera vez la responsabilidad de su alimentación, por lo cual se convierte en una etapa crítica para el desarrollo de hábitos alimentarios que repercutirán en su futura salud (Schnettler et al, 2013).

Viendo de ese modo que las afectaciones de salud en jóvenes son frecuentes, por no conformar hábitos alimenticios sanos, y, en cuestión de tecnologías la mayoría de los jóvenes usan perfectamente un Smartphone, un portátil, entre otros; entonces, surge la pregunta ¿Se hace uso de las tecnologías para conocer y empezar a practicar una dieta saludable?, de allí, se considera que el propósito del proyecto de investigación es conocer sí los jóvenes estudiantes de la Universidad Francisco de Paula Santander del sector de Ocaña hacen uso de las tecnologías de la información y la comunicación para aprender sobre la correcta alimentación, es decir, responder sí estas herramientas influyen en el aprendizaje de tener una ingesta adecuada de alimentos como beneficio para su salud futura.

\section{Reflexión Teórica}

Teoría de las Representaciones Sociales y la teoría de las necesidades de Maslow sustentan que el desarrollo que tienen los seres humanos en su entorno está orientado por necesidades básicas y estas varían dependiendo de la manera de pensar y creer que tiene cada persona, en cuestión de la alimentación cada persona presenta un nivel de interés diferente en cuanto a cuanta importancia le da a su manera de alimentarse, que tan saludable es o si no le brinda ninguna importancia, actualmente en los jóvenes puede verse una motivación por mejorar su condición física, el cuidado de su salud, imagen, entre otros; van al gimnasio, crean rutinas de ejercicio, alimentación, actividades para cuidados corporales y muchos otros que con la ayuda e información que muestran los sitios web, aplicaciones, entre muchos otros medios.

Finalmente, desde la teoría de la alimentación saludable, el proceso se logra combinando varios alimentos en forma equilibrada, ateniendo las necesidades nutritivas para un considerado crecimiento donde pueda desarrollar las capacidades físicas e intelectuales de las personas (Hernández, Armenteros, Borrell, Lancés, et al., 2004).

\section{Metodología}

La investigación de enfoque cuantitativodescriptivo ya que: "Pretenden establecer las causas de los sucesos o fenómenos que se estudian." (Sampieri, 2014, p.95) con base en la información que se recolectó de los estudiantes de la UFPS seccional Ocaña y su respectivo análisis permitieron responder que tanto inciden las TIC en la cultura de la alimentación saludable en los jóvenes. Por otra parte, la población tomada como objeto de estudio fueron los estudiantes de la Universidad Francisco de Paula Santander seccional Ocaña que se encuentran realizando 
sus estudios universitarios en distintas facultades de la misma.

Tomando como base la población estudiantil total de la universidad se realizó un muestreo probabilístico por medio de la fórmula estadística para poblaciones finitas, la cual permitió obtener una muestra de 300 estudiantes. Como técnica se empleó la técnica de la encuesta que fue dirigida a los estudiantes de la Universidad Francisco de Paula Santander Ocaña con el objetivo de obtener la información directamente de ellos y que esta sea lo más verídica posible. Una vez se obtuvo toda la información requerida en las encuestas, se pasó al procesamiento de los datos obtenidos y a su respectivo análisis por medio de tablas y figuras a través de las respuestas obtenidas logrando así, el cumplimiento de los objetivos de la investigación.

\section{Resultados}

Se investigó en los jóvenes universitarios acerca de las TICS, para conocer la relación e incidencia de las tecnologías en la nutrición y alimentación.

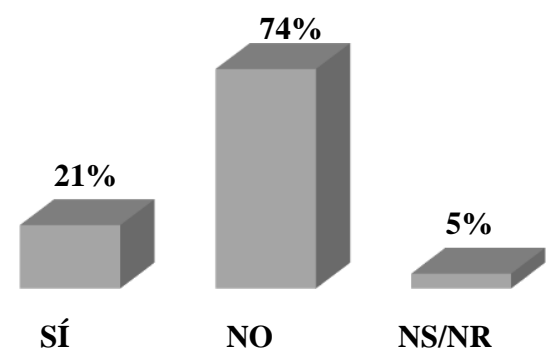

Figura 1. Conocimiento sobre sitios web de nutrición y alimentación.

Con el interés de evaluar el conocimiento que tenían los estudiantes sobre aplicaciones o sitios en internet para formarse en nutrición, el $74 \%$ respondió que no hacen uso de las plataformas para conocer sobre el estilo de vida saludable en función del consumo y, un $21 \%$ respondió afirmativamente, buscando los sitios web o aplicaciones para aprender sobre nutrición, algunos mencionaron: Redes sociales como Facebook, aplicaciones como Reto 30, Butt And Legs 21, Tuprote.com, Fastfitness, programas de cocina gymworknow, nutrivida y nutrisalud, entre otras.

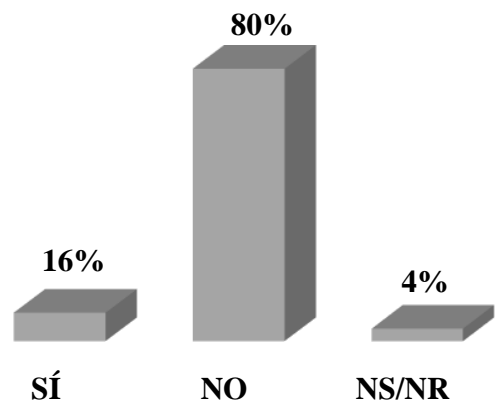

Figura 2. Manejo de una alimentación y nutrición adecuada con el uso de las TIC.

De acuerdo a los datos obtenidos, los estudiantes de la Universidad, no maneja una alimentación promovida desde las tecnologías, si bien es claro, existe una cultura no favorable sobre la nutrición, donde las posibles causas sean las condiciones del económicas o las costumbres alimenticias de la región.

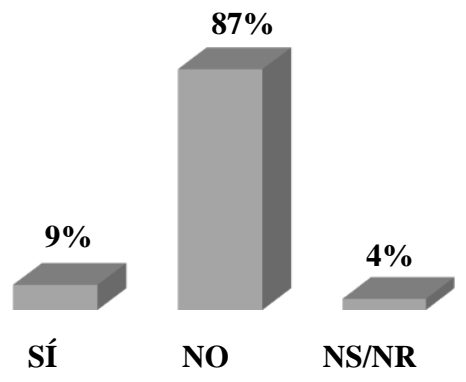

Figura 3. Interés de los estudiantes para formarse en nutrición desde las TIC. 
La Teoría de las Necesidades de Maslow menciona que las necesidades de amor afecto y pertenencia las cuales hacen referencia a el nivel de satisfacción sobre bienestar y seguridad. Apoyando los resultados obtenidos por la encuesta se cuestionaba a los estudiantes sobre el interés del uso de sitios virtuales para conocer el mundo de educación nutricional, presentándose que un $87 \%$ no acudiría a ninguno sitio web para formarse y un $9 \%$ considera favorable a través de videos de expertos nutricionistas, sitios de cocina web, entre otros.

\section{Conclusiones}

Hoy en día con la llegada de las nuevas tecnologías y la globalización todos pueden está conectados a una red que brinda información acerca de cualquier tema de interés, es por ello que se puede ver en la juventud un uso frecuente de dispositivos móviles que sirven para mejorar la comunicación, las actividades de esparcimiento para tiempo ocio, etc. Sin embargo, no existe una cultura de alimentación desde la apropiación virtual, pues bien, con base en el conocimiento de sitios web con información sobre nutrición y alimentación, se puede mostrar un gran porcentaje de jóvenes estudiantes que no tienen conocimiento y no realizan prácticas considerables de alimentación pensando en la salud y en el bienestar propio.

Del mismo modo, se puede determinar el desinterés de la comunidad estudiantil para apropiar conocimiento a través del aprovechamiento de las tecnologías para mejorar su calidad de vida y recibir orientaciones sobre nutrición balanceada, más cuando, la comunidad de estudio acude a las denominadas "comidas rápidas".

\section{Referencias}

Guerrero Bellido, D. (2006). Bases científicas de una alimentación saludable. REVISTA MEDICA UNIV NAVARRA, 50(4), 7-14.

Hernández Sampieri, R. (2014). Metodología de la investigación sexta edición. 634 .

Izquierdo Hernández, Amada, Armenteros Borrell, Mercedes, Lancés Cotilla, Luisa, y Martín González, Isabel. (2004). Alimentación saludable. Revista Cubana de Enfermería, 20(1).

Ortiz Moncada, M. d. (2017). Alimentación y nutrición. Universidad del Atlántico (Colombia).

Schnettler, B., Denegri, M., Miranda, H., Sepúlveda, J., Orellana, L., Paiva, G., y Grunert, K. (2013). Hábitos alimentarios y bienestar subjetivo en estudiantes.

Doi:10.3305/nh.2013.28.6.6751 